\title{
Color Neighborhood Union Conditions for Long Heterochromatic Paths in Edge-Colored Graphs *
}

\author{
He Chen and Xueliang Li \\ Center for Combinatorics and LPMC-TJKLC \\ Nankai University, Tianjin 300071, China \\ lxl@nankai.edu.cn
}

Submitted: Apr 12, 2007; Accepted: Nov 1, 2007; Published: Nov 12, 2007

Mathematics Subject Classifications: 05C38, 05C15

\begin{abstract}
Let $G$ be an edge-colored graph. A heterochromatic (rainbow, or multicolored) path of $G$ is such a path in which no two edges have the same color. Let $C N(v)$ denote the color neighborhood of a vertex $v$ of $G$. In a previous paper, we showed that if $|C N(u) \cup C N(v)| \geq s$ (color neighborhood union condition) for every pair of vertices $u$ and $v$ of $G$, then $G$ has a heterochromatic path of length at least $\left\lfloor\frac{2 s+4}{5}\right\rfloor$. In the present paper, we prove that $G$ has a heterochromatic path of length at least $\left\lceil\frac{s+1}{2}\right\rceil$, and give examples to show that the lower bound is best possible in some sense.
\end{abstract}

Keywords: edge-colored graph, color neighborhood, heterochromatic (rainbow, or multicolored) path.

\section{Introduction}

We use Bondy and Murty [3] for terminology and notations not defined here and consider simple graphs only.

Let $G=(V, E)$ be a graph. By an edge-coloring of $G$ we will mean a function $C: E \rightarrow$ $\mathbb{N}$, the set of natural numbers. If $G$ is assigned such a coloring, then we say that $G$ is an edge-colored graph. Denote the edge-colored graph by $(G, C)$, and call $C(e)$ the color of the edge $e \in E$. We say that $C(u v)=\emptyset$ if $u v \notin E(G)$ for $u, v \in V(G)$. For a subgraph $H$ of $G$, we denote $C(H)=\{C(e) \mid e \in E(H)\}$ and $c(H)=|C(H)|$. For a vertex $v$ of $G$, the color neighborhood $C N(v)$ of $v$ is defined as the set $\{C(e) \mid e$ is incident with $v\}$ and the color degree is $d^{c}(v)=|C N(v)|$. A path is called heterochromatic (rainbow, or

\footnotetext{
*Research supported by NSFC, PCSIRT and the "973" program.
} 
multicolored) if any two edges of it have different colors. If $u$ and $v$ are two vertices on a path $P, u P v$ denotes the segment of $P$ from $u$ to $v$, whereas $v P^{-1} u$ denotes the same segment but from $v$ to $u$.

There are many existing literature dealing with the existence of paths and cycles with special properties in edge-colored graphs. In [6], the authors showed that for a 2-edgecolored graph $G$ and three specified vertices $x, y$ and $z$, to decide whether there exists a color-alternating path from $x$ to $y$ passing through $z$ is NP-complete. The heterochromatic Hamiltonian cycle or path problem was studied by Hahn and Thomassen [10], Rödl and Winkler (see [9]), Frieze and Reed [9], and Albert, Frieze and Reed [1]. For more references, see $[2,7,8,11,12]$. Many results in these papers were proved by using probabilistic methods.

Suppose $|C N(u) \cup C N(v)| \geq s$ (color neighborhood union condition) for every pair of vertices $u$ and $v$ of $G$. In [4], the authors showed that $G$ has a heterochromatic path of length at least $\left\lceil\frac{s}{3}\right\rceil+1$. In [5], we proved that $G$ has a heterochromatic path of length at least $\left\lfloor\frac{2 s+4}{5}\right\rfloor$. In the present paper, we prove that $G$ has a heterochromatic path of length at least $\left\lceil\frac{s+1}{2}\right\rceil$, and give examples to show that the lower bound is best possible in some sense.

\section{Long heterochromatic paths for $s \leq 7$}

First, we consider the case when $1 \leq s \leq 7$, which will serve as the induction initial for our main result Theorem 3.6 in next section.

Lemma 2.1 Let $G$ be an edge-colored graph and $1 \leq s \leq 7$ an integer. Suppose that $|C N(u) \cup C N(v)| \geq s$ for every pair of vertices $u$ and $v$ of $G$. Then $G$ has a heterochromatic path of length at least $\left\lceil\frac{s+1}{2}\right\rceil$.

Proof. (1) $s=1$.

Then any edge in $G$ is a heterochromatic path of length $1=\left\lceil\frac{s+1}{2}\right\rceil$.

(2) $s=2$.

Let $e=u v$ be an arbitrary edge in $G$.

Since $|C N(u) \cup C N(v)| \geq s=2$, there exists a $v^{\prime} \in V(G)-\{u, v\}$ such that $v^{\prime} u \in E(G)$ and $C\left(v^{\prime} u\right) \neq C(u v)$, or $v^{\prime} v \in E(G)$ and $C\left(v^{\prime} v\right) \neq C(u v)$.

If $v^{\prime} u \in E(G)$ and $C\left(v^{\prime} u\right) \neq C(u v)$, then $v^{\prime} u v$ is a heterochromatic path of length $2=\left\lceil\frac{s+1}{2}\right\rceil$.

If $v^{\prime} v \in E(G)$ and $C\left(v^{\prime} v\right) \neq C(u v)$, then $v^{\prime} v u$ is a heterochromatic path of length $2=\left\lceil\frac{s+1}{2}\right\rceil$.

(3) $s=3$.

Since $|C N(u) \cup C N(v)| \geq s=3>2$ for every pair of vertices $u$ and $v$ of $G$, there is a heterochromatic path of length $2=\left\lceil\frac{s+1}{2}\right\rceil$ in $G$.

(4) $s=4$.

Since $|C N(u) \cup C N(v)| \geq s=4>2$ for every pair of vertices $u$ and $v$ of $G$, there is a 
heterochromatic path of length 2 , let $u_{0} u_{1} u_{2}$ be such a path.

Since $\left|C N\left(u_{0}\right) \cup C N\left(u_{2}\right)\right| \geq 4$, there exists a $v \in V(G)-\left\{u_{0}, u_{1}, u_{2}\right\}$ such that $C\left(v u_{0}\right) \notin\left\{C\left(u_{0} u_{1}\right), C\left(u_{1} u_{2}\right)\right\}$ or $C\left(v u_{2}\right) \notin\left\{C\left(u_{0} u_{1}\right), C\left(u_{1} u_{2}\right)\right\}$.

If $C\left(v u_{0}\right) \notin\left\{C\left(u_{0} u_{1}\right), C\left(u_{1} u_{2}\right)\right\}$, then $v u_{0} u_{1} u_{2}$ is a heterochromatic path of length $3=\left\lceil\frac{s+1}{2}\right\rceil$.

If $C\left(v u_{2}\right) \notin\left\{C\left(u_{0} u_{1}\right), C\left(u_{1} u_{2}\right)\right\}$, then $u_{0} u_{1} u_{2} v$ is a heterochromatic path of length $3=\left\lceil\frac{s+1}{2}\right\rceil$.

(5) $s=5$.

Since $|C N(u) \cup C N(v)| \geq s=5>4$ for every pair of vertices $u$ and $v$ of $G$, there is a heterochromatic path of length $3=\left\lceil\frac{s+1}{2}\right\rceil$ in $G$.

(6) $s=6$.

Since $|C N(u) \cup C N(v)| \geq s=6>4$ for every pair of vertices $u$ and $v$ of $G$, there is a heterochromatic path of length 3 , let $P=u_{0} u_{1} u_{2} u_{3}$ be such a path.

If there exists a $v \in V(G)-\left\{u_{0}, u_{1}, u_{2}, u_{3}\right\}$ such that $C\left(v u_{0}\right) \notin C(P)$ or $C\left(v u_{3}\right) \notin C(P)$, then $v u_{0} u_{1} u_{2} u_{3}$ or $u_{0} u_{1} u_{2} u_{3} v$ is a heterochromatic path of length $4=\left\lceil\frac{s+1}{2}\right\rceil$.

Otherwise, $\left|C\left(u_{0} u_{2}, u_{0} u_{3}, u_{1} u_{3}\right)-C(P)\right|=3$, since $\left|C N\left(u_{0}\right) \cup C N\left(u_{3}\right)-C(P)\right| \geq$ $\left|C N\left(u_{0}\right) \cup C N\left(u_{3}\right)\right|-|C(P)| \geq 6-3=3$. On the other hand, since $\left|C N\left(u_{0}\right) \cup C N\left(u_{3}\right)\right| \geq 6$, there exists a $v \in V(G)-\left\{u_{0}, u_{1}, u_{2}, u_{3}\right\}$ such that $C\left(v u_{0}\right)=C\left(u_{1} u_{2}\right)$ or $C\left(v u_{3}\right)=$ $C\left(u_{1} u_{2}\right)$, then $v u_{0} u_{1} u_{3} u_{2}$ or $v u_{3} u_{2} u_{0} u_{1}$ is a heterochromatic path of length $4=\left\lceil\frac{s+1}{2}\right\rceil$.

(7) $s=7$.

Since $|C N(u) \cup C N(v)| \geq s=7>6$ for every pair of vertices $u$ and $v$ of $G$, there is a heterochromatic path of length $4=\left\lceil\frac{s+1}{2}\right\rceil$ in $G$.

\section{Long heterochromatic paths for all $s \geq 1$}

In this section we will give a best possible lower bound for the length of the longest heterochromatic path in $G$ when $s \geq 7$. First, we will do some preparations.

Lemma 3.1 Suppose $P=u_{0} u_{1} u_{2} \ldots u_{l}$ is a heterochromatic path of length $l \geq 4, u_{0} u_{l} \in$ $E(G)$ and $C\left(u_{0} u_{l}\right) \notin C(P)$. If there exists a $v \in N\left(u_{0}\right)-V(P)$ such that $C\left(u_{0} v\right)=$ $C\left(u_{i-1} u_{i}\right)$ for some $1 \leq i \leq l$ that satisfies $\mid\left\{C\left(u_{i-1} w\right): w \in V(P)\right\} \cup\left\{C\left(u_{l} w\right): w \in\right.$ $V(P)\}-C(P)-C\left(u_{0} u_{l}\right) \mid \geq l-1$, then there is a heterochromatic path of length $l+1$ in $G$.

Proof. Let $C_{0}=C(P) \cup C\left(u_{0} u_{l}\right)$.

We distinguish the following 5 cases:

Case 1. $i=1$

Then $v u_{0} u_{l} P^{-1} u_{1}$ is a heterochromatic path of length $l+1$.

Case 2. $i=2$

Let

$$
\begin{aligned}
& X=\left\{3 \leq j \leq l-1: C\left(u_{1} u_{j}\right) \notin C_{0}\right\}, \\
& Y=\left\{3 \leq j \leq l-1: C\left(u_{j-1} u_{l}\right) \notin C_{0} \cup\left\{C\left(u_{1} u_{j}: j \in X\right)\right\}\right\} .
\end{aligned}
$$


Then we have

$$
\begin{gathered}
\left\{C\left(u_{1} w\right): w \in V(P)\right\}-C_{0}=\cup_{i=3}^{l} C\left(u_{1} u_{i}\right)-C_{0}=\left\{C\left(u_{1} u_{j}\right): j \in X\right\} \cup\left(C\left(u_{1} u_{l}\right)-C_{0}\right), \\
\begin{aligned}
\left\{C\left(u_{l} w\right): w \in V(P)\right\} & -C_{0}-\left\{C\left(u_{1} u_{j}\right): j \in X\right\} \\
& =\cup_{j=1}^{l-1} C\left(u_{l} u_{j-1}\right)-C_{0}-\left\{C\left(u_{1} u_{j}\right): j \in X\right\} \\
& \subseteq\left\{C\left(u_{l} u_{j-1}\right): j \in Y\right\} \cup\left(C\left(u_{1} u_{l}\right)-C_{0}\right) .
\end{aligned}
\end{gathered}
$$

So

$$
\begin{aligned}
\left\{C\left(u_{1} w\right): w \in V(P)\right\} & \cup\left\{C\left(u_{l} w\right): w \in V(P)\right\}-C_{0} \\
& \subseteq\left\{C\left(u_{1} u_{j}\right): j \in X\right\} \cup\left\{C\left(u_{l} u_{j-1}\right): j \in Y\right\} \cup\left(C\left(u_{1} u_{l}\right)-C_{0}\right) .
\end{aligned}
$$

If $C\left(u_{1} u_{l}\right) \notin C_{0}$, then $v u_{0} u_{1} u_{l} P^{-1} u_{2}$ is a heterochromatic path of length $l+1$.

Otherwise, we have $C\left(u_{1} u_{l}\right) \in C_{0}$, then

$$
\begin{aligned}
l-1 & \leq\left|\left\{C\left(u_{1} w\right): w \in V(P)\right\} \cup\left\{C\left(u_{l} w\right): w \in V(P)\right\}-C_{0}\right| \\
& \leq\left|\left\{C\left(u_{1} u_{j}\right): j \in X\right\}\right|+\left|\left\{C\left(u_{l} u_{j-1}\right): j \in Y\right\}\right| \\
& \leq|X|+|Y| .
\end{aligned}
$$

On the other hand, $X, Y \subseteq\{3, \ldots, l-1\}$, and $|\{3, \ldots, l-1\}|=l-3$, so $|X|+|Y| \geq$ $|\{3, \ldots, l-1\}|+1$. Then we can conclude that there exists a $j \in X \cap Y$. In this case, $v u_{0} u_{1} u_{j} P u_{l} u_{j-1} P^{-1} u_{2}$ is a heterochromatic path of length $l+1$.

So there exists a heterochromatic path of length $l+1$ if $i=2$.

Case 3. $i=l$

Let

$$
\begin{aligned}
& X=\left\{1 \leq j \leq l-2: C\left(u_{j-1} u_{l-1}\right) \notin C_{0}\right\} \\
& Y=\left\{1 \leq j \leq l-2: C\left(u_{j} u_{l}\right) \notin C_{0} \cup\left\{C\left(u_{j-1} u_{l-1}\right): j \in X\right\}\right\}
\end{aligned}
$$

Then

$$
\begin{aligned}
\left\{C\left(u_{l-1} w\right): w \in V(P)\right\} & \cup\left\{C\left(u_{l} w\right): w \in V(P)\right\}-C_{0} \\
& \subseteq\left\{C\left(u_{l-1} u_{j-1}\right): j \in X\right\} \cup\left\{C\left(u_{l} u_{j}\right): j \in Y\right\} .
\end{aligned}
$$

So

$$
\begin{aligned}
l-1 & \leq\left|\left\{C\left(u_{l-1} w\right): w \in V(P)\right\} \cup\left\{C\left(u_{l} w\right): w \in V(P)\right\}-C_{0}\right| \\
& \leq\left|\left\{C\left(u_{l-1} u_{j-1}\right): j \in X\right\} \cup\left\{C\left(u_{l} u_{j}\right): j \in Y\right\}\right| \\
& \leq|X|+|Y| .
\end{aligned}
$$

Since $X, Y \subseteq\{1,2, \ldots, l-2\}$ and $|\{1,2, \ldots, l-2\}|=l-2$, there exists a $j \in X \cap Y$. In this case, $v u_{0} P u_{j-1} u_{l-1} P^{-1} u_{j} u_{l}$ is a heterochromatic path of length $l+1$.

So there exists a heterochromatic path of length $l+1$ if $i=l$.

Case 4. $i=l-1$

Let

$$
\begin{aligned}
& X=\left\{1 \leq j \leq l-3: C\left(u_{j-1} u_{l-2}\right) \notin C_{0}\right\} \\
& Y=\left\{1 \leq j \leq l-3: C\left(u_{j} u_{l}\right) \notin C_{0} \cup\left\{C\left(u_{l-2} u_{j-1}\right): j \in X\right\}\right\}
\end{aligned}
$$


Then we have

$$
\begin{aligned}
\left\{C\left(u_{l-2} w\right): w \in V(P)\right\}- & C_{0}=\cup_{j=1}^{l-3} C\left(u_{j-1} u_{l-2}\right) \cup C\left(u_{l-2} u_{l}\right)-C_{0} \\
& =\left\{C\left(u_{j-1} u_{l-2}\right): j \in X\right\} \cup\left(C\left(u_{l-2} u_{l}\right)-C_{0}\right), \\
\left\{C\left(u_{l} w\right): w \in V(P)\right\} & -C_{0}-\left\{C\left(u_{j-1} u_{l-2}\right): j \in X\right\} \\
& =\cup_{j=0}^{l-2} C\left(u_{l} u_{j}\right)-C_{0}-\left\{C\left(u_{j-1} u_{l-2}\right): j \in X\right\} \\
& \subseteq\left\{C\left(u_{l} u_{j}\right): j \in Y\right\} \cup\left(C\left(u_{l-2} u_{l}\right)-C_{0}\right) .
\end{aligned}
$$

So

$$
\begin{aligned}
\left\{C\left(u_{l-2} w\right): w \in V(P)\right\} & \cup\left\{C\left(u_{l} w\right): w \in V(P)\right\}-C_{0} \\
& \subseteq\left\{C\left(u_{j-1} u_{l-2}\right): j \in X\right\} \cup\left\{C\left(u_{l} u_{j}\right): j \in Y\right\} \cup\left(C\left(u_{l-2} u_{l}\right)-C_{0}\right) .
\end{aligned}
$$

If $C\left(u_{l-2} u_{l}\right) \notin C_{0}, v u_{0} P u_{l-2} u_{l} u_{l-1}$ is a heterochromatic path of length $l+1$.

Otherwise, we have $C\left(u_{l-2} u_{l}\right) \in C_{0}$, then

$$
\begin{aligned}
l-1 & \leq\left|\left\{C\left(u_{l-2} w\right): w \in V(P)\right\} \cup\left\{C\left(u_{l} w\right): w \in V(P)\right\}-C_{0}\right| \\
& \leq\left|\left\{C\left(u_{j-1} u_{l-2}\right): j \in X\right\} \cup\left\{C\left(u_{l} u_{j}\right): j \in Y\right\}\right| \\
& \leq|X|+|Y| .
\end{aligned}
$$

Now we can conclude that there exists a $j \in X \cap Y$, since $|X|+|Y| \geq l-1>$ $|\{1, \ldots, l-3\}|+1$ and $X, Y \subseteq\{1,2, \ldots, l-3\}$. In this case, $v u_{0} P u_{j-1} u_{l-2} P^{-1} u_{j} u_{l} u_{l-1}$ is a heterochromatic path of length $l+1$.

So there exists a heterochromatic path of length $l+1$ if $i=l-1$.

Case 5. $3 \leq i \leq l-2$

Then we have $l \geq 5$.

Let

$$
\begin{aligned}
& X_{1}=\left\{1 \leq j \leq i-2: C\left(u_{i-1} u_{j-1}\right) \notin C_{0}\right\}, \\
& X_{2}=\left\{i+1 \leq j \leq l-1: C\left(u_{i-1} u_{j}\right) \notin C_{0}\right\}, \\
& \left.C_{1}=\left\{C\left(u_{i-1} u_{j-1}\right): j \in X_{1}\right\} \cup\left\{C\left(u_{i-1} u_{j}\right): j \in X_{2}\right\}\right\} \\
& Y_{1}=\left\{1 \leq j \leq i-2: C\left(u_{l} u_{j}\right) \notin C_{0} \cup C_{1}\right\}, \\
& Y_{2}=\left\{i+1 \leq j \leq l-1: C\left(u_{l} u_{j-1}\right) \notin C_{0} \cup C_{1}\right\} .
\end{aligned}
$$

Then

$$
\begin{aligned}
\left\{C\left(u_{i-1} w\right)\right. & : w \in V(P)\}-C_{0} \\
& =\left(\cup_{j=1}^{i-2} C\left(u_{i-1} u_{j-1}\right)\right) \cup\left(\cup_{j=i+1}^{l} C\left(u_{i-1} u_{j}\right)\right)-C_{0} \\
& \subseteq\left\{C\left(u_{i-1} u_{j-1}\right): j \in X_{1}\right\} \cup\left\{C\left(u_{i-1} u_{j}\right): j \in X_{2}\right\} \cup\left(C\left(u_{i-1} u_{l}\right)-C_{0}\right) \\
& =C_{1} \cup\left(C\left(u_{i-1} u_{l}\right)-C_{0}\right), \\
\left\{C\left(u_{l} w\right)\right. & : w \in V(P)\}-C_{0}-C_{1} \\
& =\left(\cup_{j=0}^{i-2} C\left(u_{j} u_{l}\right)\right) \cup C\left(u_{i-1} u_{l}\right) \cup\left(\cup_{j=i+1}^{l-1} C\left(u_{j-1} u_{l}\right)\right)-C_{0}-C_{1} \\
& \subseteq\left\{C\left(u_{l} u_{j}\right): j \in Y_{1}\right\} \cup\left\{C\left(u_{l} u_{j-1}\right): j \in Y_{2}\right\} \cup\left(C\left(u_{i-1} u_{l}\right)-C_{0}\right) .
\end{aligned}
$$


So

$$
\begin{aligned}
\left\{C\left(u_{i-1} w\right)\right. & : w \in V(P)\} \cup\left\{C\left(u_{l} w\right): w \in V(P)\right\}-C_{0} \\
\subseteq & C_{1} \cup\left\{C\left(u_{l} u_{j}\right): j \in Y_{1}\right\} \cup\left\{C\left(u_{l} u_{j-1}\right): j \in Y_{2}\right\} \cup\left(C\left(u_{i-1} u_{l}\right)-C_{0}\right) \\
= & \left\{C\left(u_{i-1} u_{j-1}\right): j \in X_{1}\right\} \cup\left\{C\left(u_{i-1} u_{j}\right): j \in X_{2}\right\} \\
& \cup\left\{C\left(u_{l} u_{j}\right): j \in Y_{1}\right\} \cup\left\{C\left(u_{l} u_{j-1}\right): j \in Y_{2}\right\} \cup\left(C\left(u_{i-1} u_{l}\right)-C_{0}\right) .
\end{aligned}
$$

If $C\left(u_{i-1} u_{l}\right) \notin C_{0}$, then $v u_{0} P u_{i-1} u_{l} P^{-1} u_{i}$ is a heterochromatic path of length $l+1$. Otherwise, we have $C\left(u_{i-1} u_{l}\right) \in C_{0}$, then

$$
\begin{aligned}
l-1 \leq & \left|\left\{C\left(u_{i-1} w\right): w \in V(P)\right\} \cup\left\{C\left(u_{l} w\right): w \in V(P)\right\}-C_{0}\right| \\
\leq & \mid\left\{C\left(u_{i-1} u_{j-1}\right): j \in X_{1}\right\} \cup\left\{C\left(u_{i-1} u_{j}\right): j \in X_{2}\right\} \\
& \cup\left\{C\left(u_{l} u_{j}\right): j \in Y_{1}\right\} \cup\left\{C\left(u_{l} u_{j-1}\right): j \in Y_{2}\right\} \mid \\
\leq & \left|X_{1}\right|+\left|X_{2}\right|+\left|Y_{1}\right|+\left|Y_{2}\right| .
\end{aligned}
$$

Since $X_{1}, Y_{1} \subseteq\{1, \ldots, i-2\}, X_{2}, Y_{2} \subseteq\{i+1, \ldots, l-1\}$, and $l-1>\mid\{1, \ldots, i-2\} \cup$ $\{i+1, \ldots, l-1\} \mid+1$, we can conclude that there exists a $j \in\left(X_{1} \cap Y_{1}\right) \cup\left(X_{2} \cap Y_{2}\right)$. If $j \in X_{1} \cap Y_{1}$, then $v u_{0} P u_{j-1} u_{i-1} P^{-1} u_{j} u_{l} P^{-1} u_{i}$ is a heterochromatic path of length $l+1$. If $j \in X_{2} \cap Y_{2}$, then $v u_{0} P u_{i-1} u_{j} P u_{l} u_{j-1} P^{-1} u_{i}$ is a heterochromatic path of length $l+1$.

So there exists a heterochromatic path of length $l+1$ if $3 \leq i \leq l-2$.

From all the cases above, we can conclude that if all the conditions in the lemma are satisfied, there exists a heterochromatic path of length $l+1$ in $G$.

Lemma 3.2 Suppose $P=u_{0} u_{1} \ldots u_{l}$ is a heterochromatic path of length $l(l \geq 4)$, $C\left(u_{0} u_{l}\right) \in C(P), 2 \leq i_{0} \leq l-1$ and $\left|\left\{C\left(u_{0} u_{i_{0}}\right), C\left(u_{i_{0}-1} u_{l}\right)\right\}-C(P)\right|=2$. If there exists a $v \in N\left(u_{0}\right)-V(P)$ such that $C\left(u_{0} v\right)=C\left(u_{i-1} u_{i}\right)$ for some $1 \leq i \leq i_{0}-1$ and $\left|\left\{C\left(u_{i-1} w\right): w \in V(P)\right\} \cup\left\{C\left(u_{l} w\right): w \in V(P)\right\}-C(P)-C\left(u_{0} u_{i_{0}}\right)-C\left(u_{i_{0}-1} u_{l}\right)\right| \geq l-2$, then there is a heterochromatic path of length $l+1$ in $G$.

Proof. Let $C_{0}=C(P) \cup C\left(u_{0} u_{i_{0}}\right) \cup C\left(u_{i_{0}-1} u_{l}\right)$.

We distinguish the following three cases:

Case 1. $i=1$

Then $v u_{0} u_{i_{0}} P u_{l} u_{i_{0}-1} P^{-1} u_{1}$ is a heterochromatic path of length $l+1$.

Case 2. $i=2$

Let

$$
\begin{aligned}
& X=\left\{j: 3 \leq j \leq l-1, j \neq i_{0}, C\left(u_{1} u_{j}\right) \notin C_{0}\right\} \\
& Y=\left\{j: 3 \leq j \leq l-1, j \neq i_{0}, C\left(u_{j-1} u_{l}\right) \notin C_{0} \cup\left\{C\left(u_{1} u_{j}\right): j \in X\right\}\right\} .
\end{aligned}
$$

Then

$$
\begin{aligned}
\left\{C\left(u_{1} w\right): w \in V(P)\right\}-C_{0} & =\cup_{j=3}^{l} C\left(u_{1} u_{j}\right)-C_{0} \\
& =\left\{C\left(u_{1} u_{j}\right): j \in X\right\} \cup\left(C\left(u_{1} u_{i_{0}}\right)-C_{0}\right) \cup\left(C\left(u_{1} u_{l}\right)-C_{0}\right),
\end{aligned}
$$




$$
\begin{aligned}
\left\{C\left(u_{l} w\right)\right. & : w \in V(P)\}-C_{0}-\left\{C\left(u_{1} u_{j}\right): j \in X\right\} \\
& =\cup_{j=1}^{l-1} C\left(u_{j-1} u_{l}\right)-C_{0}-\left\{C\left(u_{1} u_{j}\right): j \in X\right\} \\
& =\left\{C\left(u_{j-1} u_{l}\right): j \in Y\right\} \cup\left(C\left(u_{0} u_{l}\right)-C_{0}\right) \cup\left(C\left(u_{1} u_{l}\right)-C_{0}\right) \\
& =\left\{C\left(u_{j-1} u_{l}\right): j \in Y\right\} \cup\left(C\left(u_{1} u_{l}\right)-C_{0}\right) .
\end{aligned}
$$

So

$$
\begin{aligned}
\left\{C\left(u_{1} w\right)\right. & : w \in V(P)\} \cup\left\{C\left(u_{l} w\right): w \in V(P)\right\}-C_{0} \\
& =\left\{C\left(u_{1} u_{j}\right): j \in X\right\} \cup\left\{C\left(u_{j-1} u_{l}\right): j \in Y\right\} \cup\left(C\left(u_{1} u_{i_{0}}\right)-C_{0}\right) \cup\left(C\left(u_{1} u_{l}\right)-C_{0}\right) .
\end{aligned}
$$

If $C\left(u_{1} u_{i_{0}}\right) \notin C_{0}$, then $v u_{0} u_{1} u_{i_{0}} P u_{l} u_{i_{0}-1} P^{-1} u_{2}$ is a heterochromatic path of length $l+1$.

If $C\left(u_{1} u_{l}\right) \notin C_{0}$, then $v u_{0} u_{1} u_{l} P^{-1} u_{2}$ is a heterochromatic path of length $l+1$.

Otherwise, we consider the case when $\left\{C\left(u_{1} u_{i_{0}}\right), C\left(u_{1} u_{l}\right)\right\} \subseteq C_{0}$, then

$$
\begin{aligned}
|X|+|Y| & \geq\left|\left\{C\left(u_{1} u_{j}\right): j \in X\right\} \cup\left\{C\left(u_{j-1} u_{l}\right): j \in Y\right\}\right| \\
& \geq\left|\left\{C\left(u_{1} w\right): w \in V(P)\right\} \cup\left\{C\left(u_{l} w\right): w \in V(P)\right\}-C_{0}\right| \\
& \geq l-2>l-3=\left|\left\{3, \ldots, i_{0}-1, i_{0}+1, \ldots, l-1\right\}\right|+1 .
\end{aligned}
$$

Since $X, Y \subseteq\left\{3, \ldots, i_{0}-1, i_{0}+1, \ldots, l-1\right\}$, there exists a $j \in X \cap Y$, then $v u_{0} u_{1} u_{j} P u_{l} u_{j-1}$ $P^{-1} u_{2}$ is a heterochromatic path of length $l+1$.

Case 3. $3 \leq i \leq i_{0}-1$

Let

$$
\begin{aligned}
& X_{1}=\left\{j: 1 \leq j \leq i-2, C\left(u_{i-1} u_{j-1}\right) \notin C_{0}\right\}, \\
& X_{2}=\left\{j: i+1 \leq j \leq l-1, j \neq i_{0}, C\left(u_{i-1} u_{j}\right) \notin C_{0}\right\}, \\
& C_{1}=\left\{C\left(u_{i-1} u_{j-1}\right): j \in X_{1}\right\} \cup\left\{C\left(u_{i-1} u_{j}\right): j \in X_{2}\right\}, \\
& Y_{1}=\left\{j: 1 \leq j \leq i-2, C\left(u_{j} u_{l}\right) \notin C_{0} \cup C_{1}\right\}, \\
& Y_{2}=\left\{j: i+1 \leq j \leq l-1, j \neq i_{0}, C\left(u_{j-1} u_{l}\right) \notin C_{0} \cup C_{1}\right\} .
\end{aligned}
$$

Then

$$
\begin{aligned}
\left\{C\left(u_{i-1} w\right): w \in V(P)\right\}-C_{0}=\left(\cup_{j=1}^{i-2} C\left(u_{j-1} u_{i-1}\right)\right) \cup\left(\cup_{j=i+1}^{l} C\left(u_{i-1} u_{j}\right)\right)-C_{0} \\
\quad=C_{1} \cup\left(C\left(u_{i-1} u_{i_{0}}\right)-C_{0}\right) \cup\left(C\left(u_{i-1} u_{l}\right)-C_{0}\right), \\
\left\{C\left(u_{l} w\right): w \in V(P)\right\}-C_{0}-C_{1} \\
=\left(\cup_{j=0}^{i-1} C\left(u_{j} u_{l}\right)\right) \cup\left(\cup_{j=i+1}^{l-1} C\left(u_{j-1} u_{l}\right)\right)-C_{0}-C_{1} \\
\subseteq\left\{C\left(u_{j} u_{l}\right): j \in Y_{1}\right\} \cup\left\{C\left(u_{j-1} u_{l}\right): j \in Y_{2}\right\} \\
\cup\left(\left\{C\left(u_{0} u_{l}\right), C\left(u_{i-1} u_{l}\right), C\left(u_{i_{0}-1} u_{l}\right)\right\}-C_{0}\right) \\
=\left\{C\left(u_{j} u_{l}\right): j \in Y_{1}\right\} \cup\left\{C\left(u_{j-1} u_{l}\right): j \in Y_{2}\right\} \cup\left(C\left(u_{i-1} u_{l}\right)-C_{0}\right) .
\end{aligned}
$$

So

$$
\begin{aligned}
\left\{C\left(u_{i-1} w\right)\right. & : w \in V(P)\} \cup\left\{C\left(u_{l} w\right): w \in V(P)\right\}-C_{0} \\
\subseteq & \left\{C\left(u_{i-1} u_{j-1}\right): j \in X_{1}\right\} \cup\left\{C\left(u_{i-1} u_{j}\right): j \in X_{2}\right\} \cup\left\{C\left(u_{j} u_{l}\right): j \in Y_{1}\right\} \\
& \cup\left\{C\left(u_{j-1} u_{l}\right): j \in Y_{2}\right\} \cup\left(C\left(u_{i-1} u_{i_{0}}\right)-C_{0}\right) \cup\left(C\left(u_{i-1} u_{l}\right)-C_{0}\right) .
\end{aligned}
$$


If $C\left(u_{i-1} u_{i_{0}}\right) \notin C_{0}$, then $v u_{0} P u_{i-1} u_{i_{0}} P u_{l} u_{i_{0}-1} P^{-1} u_{i}$ is a heterochromatic path of length $l+1$. If $C\left(u_{i-1} u_{l}\right) \notin C_{0}$, then $v u_{0} P u_{i-1} u_{l} P^{-1} u_{i}$ is a heterochromatic path of length $l+1$.

Otherwise, we have $\left\{C\left(u_{i-1} u_{i_{0}}\right), C\left(u_{i-1} u_{l}\right)\right\} \subseteq C_{0}$, then

$$
\begin{aligned}
\left|X_{1}\right|+\left|X_{2}\right|+ & \left|Y_{1}\right|+\left|Y_{2}\right| \\
\geq & \mid\left\{C\left(u_{i-1} u_{j-1}\right): j \in X_{1}\right\} \cup\left\{C\left(u_{i-1} u_{j}\right): j \in X_{2}\right\} \\
& \cup\left\{C\left(u_{j} u_{l}\right): j \in Y_{1}\right\} \cup\left\{C\left(u_{j-1} u_{l}\right): j \in Y_{2}\right\} \mid \\
\geq & \left|\left\{C\left(u_{i-1} w\right): w \in V(P)\right\} \cup\left\{C\left(u_{l} w\right): w \in V(P)\right\}-C_{0}\right| \\
\geq & l-2>l-3=\left|\{1, \ldots, i-2\} \cup\left\{i+1, \ldots, i_{0}-1, i_{0}+1, \ldots, l-1\right\}\right|+1 .
\end{aligned}
$$

Since $X_{1}, Y_{1} \subseteq\{1,2, \ldots, i-2\}, X_{2}, Y_{2} \subseteq\left\{i+1, \ldots, i_{0}-1, i_{0}+1, \ldots, l-1\right\}$, we can conclude that there exists a $j \in\left(X_{1} \cap Y_{1}\right) \cup\left(X_{2} \cap Y_{2}\right)$. If $j \in X_{1} \cap Y_{1}$, then $v u_{0} P u_{j-1} u_{i-1} P^{-1} u_{j} u_{l} P^{-1} u_{i}$ is a heterochromatic path of length $l+1$, otherwise $j \in X_{2} \cap Y_{2}$, and in that case $v u_{0} P u_{i-1} u_{j} P u_{l} u_{j-1} P^{-1} u_{i}$ is a heterochromatic path of length $l+1$.

From all the cases above, we can conclude that if all the conditions in this lemma are satisfied, there is a heterochromatic path of length $l+1$ in $G$.

Lemma 3.3 Suppose $P=u_{0} u_{1} \ldots u_{l}$ is a heterochromatic path of length $l(l \geq 4)$, $C\left(u_{0} u_{l}\right) \in C(P), 2 \leq i_{0} \leq l-1$ and $\left|\left\{C\left(u_{0} u_{i_{0}}\right), C\left(u_{i_{0}-1} u_{l}\right)\right\}-C(P)\right|=2$. If there exists a $v \in N\left(u_{0}\right)-V(P)$ such that $C\left(u_{0} v\right)=C\left(u_{i-1} u_{i}\right)$ for some $i_{0}+1 \leq i \leq l$, and $\left|\left\{C\left(u_{i-1} w\right): w \in V(P)\right\} \cup\left\{C\left(u_{l} w\right): w \in V(P)\right\}-C(P)-C\left(u_{0} u_{i_{0}}\right)-C\left(u_{i_{0}-1} u_{l}\right)\right| \geq l-2$, then there is a heterochromatic path of length $l+1$ in $G$.

Proof. Let $C_{0}=C(P) \cup C\left(u_{0} u_{i_{0}}\right) \cup C\left(u_{i_{0}-1} u_{l}\right)$.

We distinguish the following three cases:

Case 1. $i=l$

Let

$$
\begin{aligned}
& X=\left\{j: 1 \leq j \leq l-2, j \neq i_{0}-1, C\left(u_{j-1} u_{l-1}\right) \notin C_{0}\right\} \\
& Y=\left\{j: 1 \leq j \leq l-2, j \neq i_{0}-1, C\left(u_{j} u_{l}\right) \notin C_{0} \cup\left\{C\left(u_{j-1} u_{l-1}\right): j \in X\right\}\right\} .
\end{aligned}
$$

Then

$$
\begin{aligned}
\left\{C\left(u_{l-1} w\right)\right. & : w \in V(P)\}-C_{0} \\
& =\cup_{j=1}^{l-2} C\left(u_{j-1} u_{l-1}\right)-C_{0} \\
& =\left\{C\left(u_{j-1} u_{l-1}\right): j \in X\right\} \cup\left(C\left(u_{i_{0}-2} u_{l-1}\right)-C_{0}\right), \\
\left\{C\left(u_{l} w\right)\right. & : w \in V(P)\}-C_{0}-\left\{C\left(u_{j-1} u_{l-1}\right): j \in X\right\} \\
& =\cup_{j=0}^{l-2} C\left(u_{j} u_{l}\right)-C_{0}-\left\{C\left(u_{j-1} u_{l-1}\right): j \in X\right\} \\
& =\left\{C\left(u_{j} u_{l}\right): j \in Y\right\} \cup\left(\left\{C\left(u_{0} u_{l}\right), C\left(u_{i_{0}-1} u_{l}\right)\right\}-C_{0}-\left\{C\left(u_{j-1} u_{l-1}\right): j \in X\right\}\right) \\
& =\left\{C\left(u_{j} u_{l}\right): j \in Y\right\} .
\end{aligned}
$$


So

$$
\begin{aligned}
\left\{C\left(u_{l-1} w\right)\right. & : w \in V(P)\} \cup\left\{C\left(u_{l} w\right): w \in V(P)\right\}-C_{0} \\
& =\left\{C\left(u_{j-1} u_{l-1}\right): j \in X\right\} \cup\left\{C\left(u_{j} u_{l}\right): j \in Y\right\} \cup\left(C\left(u_{i_{0}-2} u_{l-1}\right)-C_{0}\right) .
\end{aligned}
$$

If $C\left(u_{i_{0}-2} u_{l-1}\right) \notin C_{0}$, then $v u_{0} P u_{i_{0}-2} u_{l-1} P^{-1} u_{i_{0}-1} u_{l}$ is a heterochromatic path of length $l+1$.

Otherwise, we have $C\left(u_{i_{0}-2} u_{l-1}\right) \in C_{0}$, then

$$
\begin{aligned}
|X|+|Y| & \geq\left|\left\{C\left(u_{j-1} u_{l-1}\right): j \in X\right\} \cup\left\{C\left(u_{j} u_{l}\right): j \in Y\right\}\right| \\
& \geq\left|\left\{C\left(u_{l-1} w\right): w \in V(P)\right\} \cup\left\{C\left(u_{l} w\right): w \in V(P)\right\}-C_{0}\right| \\
& \geq l-2=\left|\left\{1, \ldots, i_{0}-2, i_{0}, \ldots, l-2\right\}\right|+1 .
\end{aligned}
$$

Since $X, Y \subseteq\left\{1, \ldots, i_{0}-2, i_{0}, \ldots, l-2\right\}, X \cap Y \neq \emptyset$, i.e., there exists a $j \in X \cap Y$, then $v u_{0} P u_{j-1} u_{l-1} P^{-1} u_{j} u_{l}$ is a heterochromatic path of length $l+1$.

Case 2. $i=l-1$

Let

$$
\begin{aligned}
& X=\left\{j: 1 \leq j \leq l-3, j \neq i_{0}-1, C\left(u_{j-1} u_{l-2}\right) \notin C_{0}\right\} \\
& Y=\left\{j: 1 \leq j \leq l-3, j \neq i_{0}-1, C\left(u_{j} u_{l}\right) \notin C_{0} \cup\left\{C\left(u_{j-1} u_{l-2}\right): j \in X\right\}\right\} .
\end{aligned}
$$

Then

$$
\begin{gathered}
\left\{C\left(u_{l-2} w\right): w \in V(P)\right\}-C_{0} \\
=\left(\cup_{j=1}^{l-3} C\left(u_{j-1} u_{l-2}\right) \cup C\left(u_{l-2} u_{l}\right)-C_{0}\right) \\
=\left\{C\left(u_{j-1} u_{l-2}\right): j \in X\right\} \cup\left(C\left(u_{i_{0}-2} u_{l-2}\right)-C_{0}\right) \cup\left(C\left(u_{l-2} u_{l}\right)-C_{0}\right), \\
\left\{C\left(u_{l} w\right): w \in V(P)\right\}-C_{0}-\left\{C\left(u_{j-1} u_{l-2}\right): j \in X\right\} \\
=\cup_{j=0}^{l-2} C\left(u_{j} u_{l}\right)-C_{0}-\left\{C\left(u_{j-1} u_{l-2}\right): j \in X\right\} \\
\subseteq\left\{C\left(u_{j} u_{l}\right): j \in Y\right\} \cup\left(C\left(u_{0} u_{l}\right)-C_{0}\right) \cup\left(C\left(u_{l-2} u_{l}\right)-C_{0}\right) \cup\left(C\left(u_{i_{0}-1} u_{l}\right)-C_{0}\right) \\
=\left\{C\left(u_{j} u_{l}\right): j \in Y\right\} \cup\left(C\left(u_{l-2} u_{l}\right)-C_{0}\right) .
\end{gathered}
$$

So

$$
\begin{aligned}
& \left\{C\left(u_{l-2} w\right): w \in V(P)\right\} \cup\left\{C\left(u_{l} w\right): w \in V(P)\right\}-C_{0} \\
& \quad=\left\{C\left(u_{j-1} u_{l-2}\right): j \in X\right\} \cup\left\{C\left(u_{j} u_{l}\right): j \in Y\right\} \cup\left(\left\{C\left(u_{i_{0}-2} u_{l-2}\right), C\left(u_{l-2} u_{l}\right)\right\}-C_{0}\right) .
\end{aligned}
$$

If $C\left(u_{l-2} u_{l}\right) \notin C_{0}, v u_{0} P u_{l-2} u_{l} u_{l-1}$ is a heterochromatic path of length $l+1$. If $C\left(u_{i_{0}-2} u_{l-2}\right) \notin C_{0}$, then $v u_{0} P u_{i_{0}-2} u_{l-2} P^{-1} u_{i_{0}-1} u_{l} u_{l-1}$ is a heterochromatic path of length $l+1$.

Otherwise, we have $\left\{C\left(u_{l-2} u_{l}\right), C\left(u_{i_{0}-2} u_{l-2}\right)\right\} \subseteq C_{0}$, then

$$
\begin{aligned}
|X|+|Y| & \geq\left|\left\{C\left(u_{j-1} u_{l-2}\right): j \in X\right\} \cup\left\{C\left(u_{j} u_{l}\right): j \in Y\right\}\right| \\
& \geq\left|\left\{C\left(u_{l-2} w\right): w \in V(P)\right\} \cup\left\{C\left(u_{l} w\right): w \in V(P)\right\}-C_{0}\right| \\
& \geq l-2>l-3=\left|\left\{1, \ldots, i_{0}-2, i_{0}, \ldots, l-3\right\}\right|+1 .
\end{aligned}
$$


Since $X, Y \subseteq\left\{1, \ldots, i_{0}-2, i_{0}, \ldots, l-3\right\}, X \cap Y \neq \emptyset$, i.e., there exists a $j \in X \cap Y$, then $v u_{0} P u_{j-1} u_{l-2} P^{-1} u_{j} u_{l} u_{l-1}$ is a heterochromatic path of length $l+1$.

Case 3. $i_{0}+1 \leq i \leq l-2$

Let

$$
\begin{aligned}
& X_{1}=\left\{j: 1 \leq j \leq i-2, j \neq i_{0}-1, C\left(u_{j-1} u_{i-1}\right) \notin C_{0}\right\}, \\
& X_{2}=\left\{j: i+1 \leq j \leq l-1, C\left(u_{j} u_{i-1}\right) \notin C_{0}\right\}, \\
& C_{1}=\left\{C\left(u_{j-1} u_{i-1}\right): j \in X_{1}\right\} \cup\left\{C\left(u_{j} u_{i-1}\right): j \in X_{2}\right\}, \\
& Y_{1}=\left\{j: 1 \leq j \leq i-2, j \neq i_{0}-1, C\left(u_{j} u_{l}\right) \notin C_{0} \cup C_{1}\right\}, \\
& Y_{2}=\left\{j: i+1 \leq j \leq l-1, C\left(u_{j-1} u_{l}\right) \notin C_{0} \cup C_{1}\right\} .
\end{aligned}
$$

Then

$$
\begin{aligned}
\left\{C\left(u_{i-1} w\right)\right. & : w \in V(P)\}-C_{0} \\
& =\left(\cup_{j=1}^{i-2} C\left(u_{i-1} u_{j-1}\right)\right) \cup\left(\cup_{j=i+1}^{l} C\left(u_{i-1} u_{j}\right)\right)-C_{0} \\
& =C_{1} \cup\left(C\left(u_{i-1} u_{i_{0}-2}\right)-C_{0}\right) \cup\left(C\left(u_{i-1} u_{l}\right)-C_{0}\right), \\
\left\{C\left(u_{l} w\right)\right. & : w \in V(P)\}-C_{0}-C_{1} \\
& =\left(\cup_{j=0}^{i-2} C\left(u_{l} u_{j}\right)\right) \cup\left(C\left(u_{l} u_{i-1}\right)\right) \cup\left(\cup_{j=i+1}^{l-1} C\left(u_{j-1} u_{l}\right)\right)-C_{0}-C_{1} \\
& \subseteq\left\{C\left(u_{j} u_{l}\right): j \in Y_{1}\right\} \cup\left\{C\left(u_{j-1} u_{l}\right): j \in Y_{2}\right\} \cup\left(\left\{C\left(u_{i_{0}-1} u_{l}\right) \cup C\left(u_{i-1} u_{l}\right)\right\}-C_{0}\right) \\
& =\left\{C\left(u_{j} u_{l}\right): j \in Y_{1}\right\} \cup\left\{C\left(u_{j-1} u_{l}\right): j \in Y_{2}\right\} \cup\left(C\left(u_{i-1} u_{l}\right)-C_{0}\right) .
\end{aligned}
$$

So

$$
\begin{aligned}
\left\{C\left(u_{i-1} w\right):\right. & w \in V(P)\} \cup\left\{C\left(u_{l} w\right): w \in V(P)\right\}-C_{0} \\
\subseteq & \left\{C\left(u_{j-1} u_{i-1}\right): j \in X_{1}\right\} \cup\left\{C\left(u_{j} u_{i-1}\right): j \in X_{2}\right\} \cup\left\{C\left(u_{j} u_{l}\right): j \in Y_{1}\right\} \\
& \cup\left\{C\left(u_{j-1} u_{l}\right): j \in Y_{2}\right\} \cup\left(C\left(u_{i-1} u_{i_{0}-2}\right)-C_{0}\right) \cup\left(C\left(u_{i-1} u_{l}\right)-C_{0}\right) .
\end{aligned}
$$

If $C\left(u_{i-1} u_{i_{0}-2}\right) \notin C_{0}$, then $v u_{0} P u_{i_{0}-2} u_{i-1} P^{-1} u_{i_{0}-1} u_{l} P^{-1} u_{i}$ is a heterochromatic path of length $l+1$. If $C\left(u_{i-1} u_{l}\right) \notin C_{0}$, then $v u_{0} P u_{i-1} u_{l} P^{-1} u_{i}$ is a heterochromatic path of length $l+1$.

Otherwise, we have $\left\{C\left(u_{i-1} u_{i_{0}-2}\right), C\left(u_{l} u_{i-1}\right)\right\} \subseteq C_{0}$, then

$$
\begin{aligned}
\left|X_{1}\right|+\mid & X_{2}|+| Y_{1}|+| Y_{2} \mid \\
\geq & \mid\left\{C\left(u_{j-1} u_{i-1}\right): j \in X_{1}\right\} \cup\left\{C\left(u_{i-1} u_{j}\right): j \in X_{2}\right\} \\
& \cup\left\{C\left(u_{j} u_{l}\right): j \in Y_{1}\right\} \cup\left\{C\left(u_{j-1} u_{l}\right): j \in Y_{2}\right\} \mid \\
\geq & \left|\left\{C\left(u_{i-1} w\right): w \in V(P)\right\} \cup\left\{C\left(u_{l} w\right): w \in V(P)\right\}-C_{0}\right| \\
\geq & l-2>l-3=\left|\left\{1, \ldots, i_{0}-2, i_{0}, \ldots, i-2\right\} \cup\{i+1, \ldots, l-1\}\right|+1 .
\end{aligned}
$$

Since $X_{1}, Y_{1} \subseteq\left\{1, \ldots, i_{0}-2, i_{0}, \ldots, i-2\right\}, X_{2}, Y_{2} \subseteq\{i+1, \ldots, l-1\},\left(X_{1} \cap Y_{1}\right) \cup$ $\left(X_{2} \cap Y_{2}\right) \neq \emptyset$, i.e., there exists a $j \in\left(X_{1} \cap Y_{1}\right) \cup\left(X_{2} \cap Y_{2}\right)$. If $j \in X_{1} \cap Y_{1}$, then $v u_{0} P u_{j-1} u_{i-1} P^{-1} u_{j} u_{l} P^{-1} u_{i}$ is a heterochromatic path of length $l+1$. If $j \in X_{2} \cap Y_{2}$, then $v u_{0} P u_{i-1} u_{j} P u_{l} u_{j-1} P^{-1} u_{i}$ is a heterochromatic path of length $l+1$.

From all the cases above, we can conclude that if all the conditions in the lemma are satisfied, there exists a heterochromatic path of length $l+1$ in $G$. 
Theorem 3.4 Let $G$ be an edge-colored graph and $|C N(u) \cup C N(v)| \geq s \geq 1$ for any two vertices $u$ and $v$ in $G$. Then there exists a heterochromatic path of length $\left\lceil\frac{s+1}{2}\right\rceil$ in $G$.

Proof. We will prove the theorem by induction.

If $1 \leq s \leq 7$, our Theorem 2.1 shows that $G$ has a heterochromatic path of length at least $\left\lceil\frac{s+1}{2}\right\rceil$.

Now we shall only consider the case when $s \geq 8$. Assume that if $|C N(u) \cup C N(v)| \geq$ $s-1$ for any $u, v \in V(G), G$ has a heterochromatic path of length at least $\left\lceil\frac{(s-1)+1}{2}\right\rceil \geq$ $\left\lceil\frac{7+1}{2}\right\rceil=4$. Then we need only to show that if $|C N(u) \cup C N(v)| \geq s$ for any $u, v \in V(G)$, $G$ has a heterochromatic path of length $\left\lceil\frac{s+1}{2}\right\rceil$. Since if $s$ is odd then $\left\lceil\frac{s}{2}\right\rceil=\left\lceil\frac{s+1}{2}\right\rceil$, we need only to show that if $s$ is even, $G$ has a heterochromatic path of length at least $\left\lceil\frac{s+1}{2}\right\rceil$.

By the assumption we know that $G$ has a heterochromatic path of length at least $\left\lceil\frac{(s-1)+1}{2}\right\rceil=\left\lceil\frac{s}{2}\right\rceil$. Assume that the longest heterochromatic path in $G$ is of length $l=\left\lceil\frac{s}{2}\right\rceil$ and $P=u_{0} u_{1} \ldots u_{l}$ is such a path.

Now we will show that $N\left(u_{0}\right) \subseteq V(P)$ by contradiction. Assume $N\left(u_{0}\right)-V(P) \neq \emptyset$ and $v \in N\left(u_{0}\right)-V(P)$. Then $C\left(u_{0} v\right) \notin C(P)$ or $C\left(u_{0} v\right) \in C(P)$.

If $C\left(u_{0} v\right) \notin C(P), v u_{0} P u_{l}$ is a heterochromatic path of length $l+1$, a contradiction to the assumption that the longest heterochromatic path in $G$ is of length $l$.

Now we shall only consider the case when $C\left(u_{0} v\right)=C\left(u_{i-1} u_{i}\right)$ for some $1 \leq i \leq l$. We distinguish the following cases:

Case 1. $C\left(u_{0} u_{l}\right) \notin C(P)$

If there exists a $w \in N\left(u_{i-1}\right)-V(P)$ such that $C\left(u_{i-1} w\right) \notin C(P) \cup C\left(u_{0} u_{l}\right)$, then $w u_{i-1} P^{-1} u_{0} u_{l} P^{-1} u_{i}$ is a heterochromatic path of length $l+1$, a contradiction. So we have that $C N\left(u_{i-1}\right)-C(P)-C\left(u_{0} u_{l}\right) \subseteq\left\{C\left(u_{i-1} w\right): w \in V(P)\right\}-C(P)-C\left(u_{0} u_{l}\right)$.

On the other hand, if there exists a $w \in N\left(u_{l}\right)-V(P)$ such that $C\left(u_{l} w\right) \notin C(P)$, $u_{0} P u_{l} w$ is a heterochromatic path of length $l+1$, a contradiction. So we also have that $C N\left(u_{l}\right)-C(P) \subseteq\left\{C\left(u_{l} w\right): w \in V(P)\right\}-C(P)$, then $C N\left(u_{l}\right)-C(P)-C\left(u_{0} u_{l}\right) \subseteq$ $\left\{C\left(u_{l} w\right): w \in V(P)\right\}-C(P)-C\left(u_{0} u_{l}\right)$.

So $C N\left(u_{i-1}\right) \cup C N\left(u_{l}\right) \subseteq\left\{C\left(u_{i-1} w\right): w \in V(P)\right\} \cup\left\{C\left(u_{l} w\right): w \in V(P)\right\} \cup C(P) \cup$ $C\left(u_{0} u_{l}\right)$. Now we can get that

$$
\begin{aligned}
s \leq & \left|C N\left(u_{i-1}\right) \cup C N\left(u_{l}\right)\right| \\
\leq & \left|\left\{C\left(u_{i-1} w\right): w \in V(P)\right\} \cup\left\{C\left(u_{l} w\right): w \in V(P)\right\} \cup C(P) \cup C\left(u_{0} u_{l}\right)\right| \\
\leq & \left|\left\{C\left(u_{i-1} w\right): w \in V(P)\right\} \cup\left\{C\left(u_{l} w\right): w \in V(P)\right\}-C(P)-C\left(u_{0} u_{l}\right)\right| \\
& +|C(P)|+\left|C\left(u_{0} u_{l}\right)\right| \\
= & \left|\left\{C\left(u_{i-1} w\right): w \in V(P)\right\} \cup\left\{C\left(u_{l} w\right): w \in V(P)\right\}-C(P)-C\left(u_{0} u_{l}\right)\right|+l+1 .
\end{aligned}
$$

So $\left|\left\{C\left(u_{i-1} w\right): w \in V(P)\right\} \cup\left\{C\left(u_{l} w\right): w \in V(P)\right\}-C(P)-C\left(u_{0} u_{l}\right)\right| \geq s-l-1=$ $2 l-l-1=l-1$. Then by Lemma 3.1, there is a heterochromatic path of length $l+1$ in $G$, a contradiction.

Case 2. $C\left(u_{0} u_{l}\right) \in C(P)$

Since $P$ is one of the longest heterochromatic path in $G$, there does not exist any $w \in N\left(u_{0}\right) \cup N\left(u_{l}\right)-V(P)$ such that $C\left(u_{0} w\right) \notin C(P)$ or $C\left(u_{l} w\right) \notin C(P)$, otherwise 
$w u_{0} P u_{l}$ or $u_{0} P u_{l} w$ is a heterochromatic path of length $l+1$, a contradiction.

Let

$$
\begin{aligned}
& X=\left\{2 \leq i \leq l-1: C\left(u_{0} u_{i}\right) \notin C(P)\right\} \\
& Y=\left\{2 \leq i \leq l-1: C\left(u_{i-1} u_{l}\right) \notin C(P) \cup\left\{C\left(u_{0} u_{i}\right): i \in X\right\}\right\} .
\end{aligned}
$$

Then

$$
C N\left(u_{0}\right) \cup C N\left(u_{l}\right)-C(P) \subseteq\left\{C\left(u_{0} u_{i}\right): i \in X\right\} \cup\left\{C\left(u_{i-1} u_{l}\right): i \in Y\right\},
$$

So

$$
\begin{aligned}
|X|+|Y| & \geq\left|\left\{C\left(u_{0} u_{i}\right): i \in X\right\} \cup\left\{C\left(u_{i-1} u_{l}\right): i \in Y\right\}\right| \\
& \geq\left|C N\left(u_{0}\right) \cup C N\left(u_{l}\right)-C(P)\right| \\
& \geq\left|C N\left(u_{0}\right) \cup C N\left(u_{l}\right)\right|-|C(P)| \\
& \geq s-l=l>|\{2,3, \ldots, l-1\}|+1 .
\end{aligned}
$$

Now we can conclude that there exists an $i_{0}\left(2 \leq i_{0} \leq l-1\right)$ such that $i_{0} \in X \cap Y$, i.e., $\left|\left\{C\left(u_{0} u_{i_{0}}\right), C\left(u_{i_{0}-1} u_{l}\right)\right\}-C(P)\right|=2$, since $X, Y \subseteq\{2, \ldots, l-1\}$.

Then we distinguish the following 3 subcases:

Subcase 1. $1 \leq i \leq i_{0}-1$

If there exists a $w \in N\left(u_{i-1}\right)-V(P)$ such that $C\left(u_{i-1} w\right) \notin C(P) \cup\left\{C\left(u_{0} u_{i_{0}}\right)\right.$, $\left.C\left(u_{i_{0}-1} u_{l}\right)\right\}, w u_{i-1} P^{-1} u_{0} u_{i_{0}} P u_{l} u_{i_{0}-1} P^{-1} u_{i}$ is a heterochromatic path of length $l+1$, a contradiction. So we have that $C N\left(u_{i-1}\right)-C(P)-C\left(u_{0} u_{i_{0}}\right)-C\left(u_{i_{0}-1} u_{l}\right) \subseteq\left\{C\left(u_{i-1} w\right)\right.$ : $w \in V(P)\}-C(P)-C\left(u_{0} u_{i_{0}}\right)-C\left(u_{i_{0}-1} u_{l}\right)$.

On the other hand, if there exists a $w \in N\left(u_{l}\right)-V(P)$ such that $C\left(u_{l} w\right) \notin C(P)$, $u_{0} P u_{l} w$ is a heterochromatic path of length $l+1$, a contradiction. So we also have that $C N\left(u_{l}\right)-C(P) \subseteq\left\{C\left(u_{l} w\right): w \in V(P)\right\}-C(P)$, then $C N\left(u_{l}\right)-C(P)-C\left(u_{0} u_{i_{0}}\right)-$ $C\left(u_{i_{0}-1} u_{l}\right) \subseteq\left\{C\left(u_{l} w\right): w \in V(P)\right\}-C(P)-C\left(u_{0} u_{i_{0}}\right)-C\left(u_{i_{0}-1} u_{l}\right)$.

So

$$
\begin{aligned}
C N\left(u_{i-1}\right) \cup C N\left(u_{l}\right) \subseteq & \left\{C\left(u_{i-1} w\right): w \in V(P)\right\} \cup\left\{C\left(u_{l} w\right): w \in V(P)\right\} \cup C(P) \\
& \cup\left\{C\left(u_{0} u_{i_{0}}\right), C\left(u_{i_{0}-1} u_{l}\right)\right\} .
\end{aligned}
$$

Now we can get that

$$
\begin{aligned}
s \leq & \left|C N\left(u_{i-1}\right) \cup C N\left(u_{l}\right)\right| \\
\leq & \left|\left\{C\left(u_{i-1} w\right): w \in V(P)\right\} \cup\left\{C\left(u_{l} w\right): w \in V(P)\right\} \cup C(P) \cup\left\{C\left(u_{0} u_{i_{0}}\right), C\left(u_{i_{0}-1} u_{l}\right)\right\}\right| \\
\leq & \mid\left\{C\left(u_{i-1} w\right): w \in V(P)\right\} \cup\left\{C\left(u_{l} w\right): w \in V(P)\right\}-C(P)-C\left(u_{0} u_{i_{0}}\right) \\
& -C\left(u_{i_{0}-1} u_{l}\right)|+| C(P)|+| C\left(u_{0} u_{i_{0}}\right)|+| C\left(u_{i_{0}-1} u_{l}\right) \mid \\
= & \left|\left\{C\left(u_{i-1} w\right): w \in V(P)\right\} \cup\left\{C\left(u_{l} w\right): w \in V(P)\right\}-C(P)-C\left(u_{0} u_{i_{0}}\right)-C\left(u_{i_{0}-1} u_{l}\right)\right| \\
& +l+2 .
\end{aligned}
$$

So $\left|\left\{C\left(u_{i-1} w\right): w \in V(P)\right\} \cup\left\{C\left(u_{l} w\right): w \in V(P)\right\}-C(P)-C\left(u_{0} u_{i_{0}}\right)-C\left(u_{i_{0}-1} u_{l}\right)\right| \geq$ $s-l-2=2 l-l-2=l-2$. Then by Lemma 3.2, there is a heterochromatic path of length $l+1$ in $G$, a contradiction.

Subcase 2. $i=i_{0}$

Then $v u_{0} u_{i_{0}} P u_{l} u_{i_{0}-1} P^{-1} u_{1}$ is a heterochromatic path of length $l+1$, a contradiction. 
Subcase 3. $i_{0}+1 \leq i \leq l$

If there exists a $w \in N\left(u_{i-1}\right)-V(P)$ such that $C\left(u_{i-1} w\right) \notin C(P) \cup\left\{C\left(u_{0} u_{i_{0}}\right)\right.$, $\left.C\left(u_{i_{0}-1} u_{l}\right)\right\}, w u_{i-1} P^{-1} u_{i_{0}} u_{0} P u_{i_{0}-1} u_{l} P^{-1} u_{i}$ is a heterochromatic path of length $l+1$, a contradiction. So we have that $C N\left(u_{i-1}\right)-C(P)-C\left(u_{0} u_{i_{0}}\right)-C\left(u_{i_{0}-1} u_{l}\right) \subseteq\left\{C\left(u_{i-1} w\right)\right.$ : $w \in V(P)\}-C(P)-C\left(u_{0} u_{i_{0}}\right)-C\left(u_{i_{0}-1} u_{l}\right)$.

On the other hand, if there exists a $w \in N\left(u_{l}\right)-V(P)$ such that $C\left(u_{l} w\right) \notin C(P)$, $u_{0} P u_{l} w$ is a heterochromatic path of length $l+1$, a contradiction. So we also have that $C N\left(u_{l}\right)-C(P) \subseteq\left\{C\left(u_{l} w\right): w \in V(P)\right\}-C(P)$, then $C N\left(u_{l}\right)-C(P)-C\left(u_{0} u_{i_{0}}\right)-$ $C\left(u_{i_{0}-1} u_{l}\right) \subseteq\left\{C\left(u_{l} w\right): w \in V(P)\right\}-C(P)-C\left(u_{0} u_{i_{0}}\right)-C\left(u_{i_{0}-1} u_{l}\right)$.

So

$$
\begin{aligned}
C N\left(u_{i-1}\right) \cup C N\left(u_{l}\right) \subseteq & \left\{C\left(u_{i-1} w\right): w \in V(P)\right\} \cup\left\{C\left(u_{l} w\right): w \in V(P)\right\} \cup C(P) \\
& \cup\left\{C\left(u_{0} u_{i_{0}}\right), C\left(u_{i_{0}-1} u_{l}\right)\right\}
\end{aligned}
$$

Now we can get that

$$
\begin{aligned}
s \leq & \left|C N\left(u_{i-1}\right) \cup C N\left(u_{l}\right)\right| \\
\leq & \left|\left\{C\left(u_{i-1} w\right): w \in V(P)\right\} \cup\left\{C\left(u_{l} w\right): w \in V(P)\right\} \cup C(P) \cup\left\{C\left(u_{0} u_{i_{0}}\right), C\left(u_{i_{0}-1} u_{l}\right)\right\}\right| \\
\leq & \mid\left\{C\left(u_{i-1} w\right): w \in V(P)\right\} \cup\left\{C\left(u_{l} w\right): w \in V(P)\right\}-C(P)-C\left(u_{0} u_{i_{0}}\right) \\
& -C\left(u_{i_{0}-1} u_{l}\right)|+| C(P)|+| C\left(u_{0} u_{i_{0}}\right)|+| C\left(u_{i_{0}-1} u_{l}\right) \mid \\
= & \left.\mid\left\{C\left(u_{i-1} w\right): w \in V(P)\right\} \cup\left\{C\left(u_{l} w\right): w \in V(P)\right\}-C(P)-C\left(u_{0} u_{i_{0}}\right)-C\left(u_{i_{0}-1} u_{l}\right)\right) \mid \\
& +l+2 .
\end{aligned}
$$

So $\left|\left\{C\left(u_{i-1} w\right): w \in V(P)\right\} \cup\left\{C\left(u_{l} w\right): w \in V(P)\right\}-C(P)-C\left(u_{0} u_{i_{0}}\right)-C\left(u_{i_{0}-1} u_{l}\right)\right| \geq$ $s-l-2=2 l-l-2=l-2$. Then by Lemma 3.3, there is a heterochromatic path of length $l+1$ in $G$, a contradiction.

From all the cases above, we can conclude that if $C\left(u_{0} v\right)=C\left(u_{i-1} u_{i}\right)$ for some $1 \leq$ $i \leq l$, we will get a contradiction.

So we can conclude that $N\left(u_{0}\right) \subseteq V(P)$.

In the same way, we can also get that $N\left(u_{l}\right) \subseteq V(P)$.

Now we have

$$
C N\left(u_{0}\right) \cup C N\left(u_{l}\right)=\left\{C\left(u_{0} u_{i}\right): 1 \leq i \leq l-1\right\} \cup\left\{C\left(u_{i} u_{l}\right): 1 \leq i \leq l-1\right\} \cup C\left(u_{0} u_{l}\right) .
$$

Then

$$
\begin{aligned}
\left|C N\left(u_{0}\right) \cup C N\left(u_{l}\right)\right| & =\left|\left\{C\left(u_{0} u_{i}\right): 1 \leq i \leq l-1\right\} \cup\left\{C\left(u_{i} u_{l}\right): 1 \leq i \leq l-1\right\} \cup C\left(u_{0} u_{l}\right)\right| \\
& \leq 2|\{1,2, \ldots, l-1\}|+1=2 l-1=s-1<s,
\end{aligned}
$$

a contradiction to the assumption that $|C N(u) \cup C N(v)| \geq s$ for any $u, v \in V(G)$.

So the longest heterochromatic path is of length greater than $l$, then there must exist a heterochromatic path of length $l+1=\left\lceil\frac{s+1}{2}\right\rceil$ in $G$.

The proof is now complete.

Finally, we give examples to show that our lower bound is best possible. Let $s$ be a positive integer. If $s$ is even, let $G_{s}$ be the graph obtained from the complete graph 
$K_{\frac{s+4}{2}}$ by deleting an edge; if $s$ is odd, let $G_{s}$ be the complete graph $K_{\frac{s+3}{2}}$. Then, color the edges of $G_{s}$ by different colors for any two different edges. So, for any $s \geq 1$ we have that $|C N(u) \cup C N(v)| \geq s$ for any pair of vertices $u$ and $v$ in $G$, and any longest heterochromatic path in $G$ is of length $\left\lceil\frac{s+1}{2}\right\rceil$.

\section{References}

[1] M. Albert, A. Frieze and B. Reed, Multicolored Hamilton cycles, Electronic J. Combin. 2 (1995), $\sharp R 10$.

[2] M. Axenovich, T. Jiang and Zs. Tuza, Local anti-Ramsey numbers of graphs, Combin. Probab. Comput. 12(2003), 495-511.

[3] J.A. Bondy and U.S.R. Murty, Graph Theory with Applications, Macmillan London and Elsvier, New York (1976).

[4] H.J. Broersma, X.L. Li, G. Woeginger and S.G. Zhang, Paths and cycles in colored graphs, Australasian J. Combin. 31(2005), 297-309.

[5] H. Chen and X.L. Li, Color degree and color neighborhood union conditions for long heterochromatic paths in edge-colored graphs, arXiv:math.CO/0512144 v1 7 Dec 2005.

[6] W.S. Chou, Y. Manoussakis, O. Megalaki, M. Spyratos and Zs. Tuza, Paths through fixed vertices in edge-colored graphs, Math. Inf. Sci. Hun. 32(1994), 49-58.

[7] P. Erdős and Zs. Tuza, Rainbow Hamiltonian paths and canonically colored subgraphs in infinite complete graphs, Mathematica Pannonica 1(1990), 5-13.

[8] P. Erdős and Zs. Tuza, Rainbow subgraphs in edge-colorings of complete graphs, Ann. Discrete Math. 55(1993), 81-88.

[9] A.M. Frieze and B.A. Reed, Polychromatic Hamilton cycles, Discrete Math. 118(1993), 69-74.

[10] G. Hahn and C. Thomassen, Path and cycle sub-Ramsey numbers and edge-coloring conjecture, Discrete Math. 62(1)(1986), 29-33.

[11] Y. Manoussakis, M. Spyratos and Zs. Tuza, Cycles of given color patterns, J. Graph Theory 21(1996), 153-162.

[12] Y. Manoussakis, M. Spyratos, Zs. Tuza and M. Voigt, Minimal colorings for properly colored subgraphs, Graphs and Combin. 12(1996), 345-360. 